\title{
An unusual complication of endotracheal intubation: ingestion of a bulb in a premature neonate
}

\author{
Özmert M.A. Özdemir ${ }^{1}$, Ali Koçyiğit ${ }^{2}$, Cem Çiçek ${ }^{3}$, Uğur Koltuksuz ${ }^{4}$, Hacer Ergin ${ }^{1}$ \\ ${ }^{1}$ Division of Neonatology, ${ }^{3}$ Department of Pediatrics, Departments of ${ }^{2}$ Radiology, and ${ }^{4}$ Pediatric Surgery, Pamukkale \\ University Faculty of Medicine, Denizli, Turkey. E-mail: drozmert@gmail.com \\ Received: 19 March 2015, Revised: 8 April 2015, Accepted: 12 May 2015
}

SUMMARY: Özdemir ÖMA, Koçyiğit A, Çiçek C, Koltuksuz U, Ergin H. An unusual complication of endotracheal intubation: ingestion of a bulb in a premature neonate. Turk J Pediatr 2016; 58: 119-120.

Endotracheal intubation is a common daily procedure used for sustaining life in premature and term neonates in the neonatal intensive care unit. This procedure can be associated with some iatrogenic complications. One of these complications is ingesting a laryngoscope light bulb, and it is an extremely rare complication in neonates. The consequences of ingestion or aspiration may be serious and life-threatening. Herein, a male premature neonate, ingested a laryngoscope light bulb during endotracheal intubation because of surfactant replacement therapy, is reported. This case re-affirms the importance of checking the integrity of equipment before intubation effort.

Key words: endotracheal intubation, laryngoscope light bulb, ingestion, preterm.

Endotracheal intubation is an extremely common daily procedure, used in both the operating room for surgical intervention and for sustaining life in premature and term neonates in the neonatal intensive care unit $^{1}$. This procedure can be associated with some iatrogenic complications. One of these complications is ingesting a laryngoscope light bulb, and it is an extremely rare complication of endotracheal intubation in neonates ${ }^{2}, 3$.

Herein, a premature male infant, ingested a laryngoscope light bulb during endotracheal intubation because of surfactant replacement therapy for respiratory distress syndrome (RDS), is reported.

\section{Case Report}

A male premature neonate with birth weight $1060 \mathrm{~g}$ (10-25p) was born at 29 weeks gestation by cesarean section due to preterm labor and fetal distress. His Apgar scores were 6 and 8 at the first and 5th minute of the birth, respectively. Physical examination revealed respiratory distress symptoms including tachypnea, nasal flaring, grunting, and retraction of respiratory muscles; therefore, nasal continuous positive airway pressure (CPAP) therapy was carried out to the patient. His chest X-ray showed signs of RDS; so he was diagnosed as RDS and INSURE (INtubate-SURfactant-Extubate to CPAP) protocol was planed. After the first intubation procedure, it was noticed that the light source of the laryngoscope became nonfunctional. On inspection, the laryngoscope light bulb had disappeared and could not be located in the immediate surroundings. Hence, it was assumed that the bulb had been either aspirated or ingested by the patient. The presence of the bulb in the stomach of the patient was demonstrated by a radiograph of the chest and abdomen (Fig. 1). The bulb in the patient's stomach was also confirmed by ultrasonography (US) (Fig. 2). Interestingly, during ultrasonographic examination, we observed that the bulb passed spontaneously from the stomach to the duodenum when the patient leaned to right side position on the $6^{\text {th }}$ postnatal day. Parents of the patient were informed about this condition and written informed consent was also obtained. On the $9^{\text {th }}$ postnatal day, the bulb passed spontaneously in the patient's feces without any complication. The patient was discharged on full oral feeding and healthy one month later. 


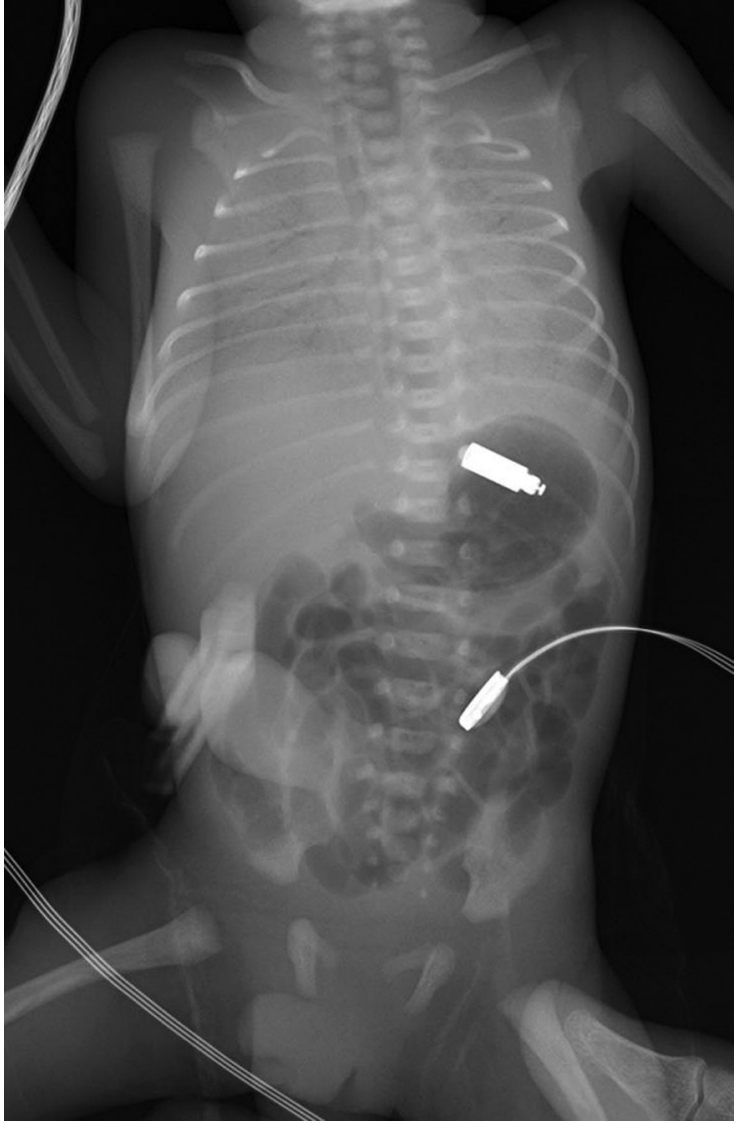

Fig. 1. X-ray of the chest and abdomen demonstrates diffuse ground-glass opacity representing respiratory distress syndrome and the foreign body (the bulb) in the stomach as well.

\section{Discussion}

To our knowledge, only two cases of ingestion of laryngoscope light bulb in the neonatal period have been previously reported in the medical literature ${ }^{2,}{ }^{3}$. Ince et al. ${ }^{3}$ reported that a female term newborn ingested a bulb after the endotracheal intubation due to suctioning of meconium staining amniotic fluid from the trachea. This patient removed the light bulb by vomiting after a few hours. Naumovski et al. ${ }^{2}$ also reported that a male newborn ingested a bulb when the patient was intubated because of meconium staining of the amniotic fluid. Then the ingested bulb passed spontaneously in the baby's feces without any complication ${ }^{2}$. To our knowledge, our patient is the first reported premature neonate ingesting a laryngoscope light bulb after the endotracheal intubation in the literature. The ingested bulb also passed spontaneously in the patient's feces without any complication. As it is shown in previous case reports, the ingested bulb of the premature

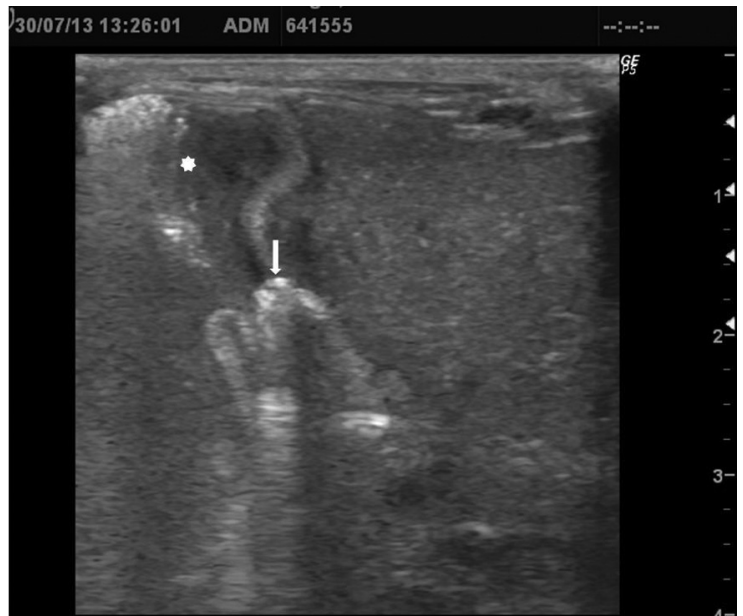

Fig. 2. Transvers ultrasound image shows the hyperechogenic bulb in the pyloric and duodenal segments (arrow). Asterix depicts the gastric antrum.

patient is demonstrated by X-ray of the chest and abdomen ${ }^{2,3}$. Furthermore, the presence of the bulb in the premature infant's stomach has been confirmed by ultrasonographic examination for the first time in the literature.

In conclusion, ingestion of a laryngoscope light bulb is an extremely rare complication in the neonatal period. It may be passed spontaneously in the premature patient's feces without any complication; however, serious and life-threatening consequences may occur if the bulb is aspirated during intubation. We recommend that fiberoptic laryngoscopes be used in every case instead of light bulb laryngoscope, and that all equipment be checked before intubation procedure.

As previously reported, this premature case report re-affirms the importance of checking the integrity of equipment before resuscitative or intubation efforts.

\section{REFERENCES}

1. Wei JL, Bond J. Management and prevention of endotracheal intubation injury in neonates. Curr Opin Otolaryngol Head Neck Surg 2011; 19: 474-477.

2. Naumovski L, Schaffer K, Fleisher B. Ingestion of a laryngoscope light bulb during delivery room resuscitation. Pediatrics 1991; 87: 581-582.

3. Ince $\mathrm{Z}$, Tuğcu D, Coban A. An unusual complication of endotracheal intubation: ingestion of a laryngoscope bulb. Pediatr Emerg Care 1998; 14: 275-276.

4. Delport SD, Gibson BH. Ingestion of a laryngoscope light bulb during tracheal intubation. S Afr Med J 1992; 81: 579 . 